\title{
Coronary artery calcification: More than meets the eye
}

\author{
Xianxi Huang, MD, ${ }^{\mathrm{a}, \mathrm{b}, \mathrm{c}}$ Jessica D’Addabbo, BS, ${ }^{\mathrm{a}, \mathrm{b}}$ and Patricia K. Nguyen, $\mathrm{MD}^{\mathrm{a}, \mathrm{b}, \mathrm{d}}$ \\ a Division of Cardiovascular Medicine, Stanford University, Stanford, CA \\ b Stanford Cardiovascular Institute, Stanford, CA \\ c The First Affiliated Hospital of Shantou University Medical College, Shantou, China \\ d Department of Veteran Affairs, Palo Alto, CA
}

Received Jan 26, 2020; accepted Jan 27, 2020

doi: $10.1007 /$ s12350-020-02058-8

\section{See related article, pp. 2207-2214}

As the clinical world moves closer toward personalized medicine, we learn once again that the devil is in the details. If we take a one-fits-all perspective, we can perhaps miss nuances that can explain why all the pieces of the puzzle do not fit perfectly for every patient. In this issue of the journal, Hsu et $\mathrm{al}^{1}$ use computed tomographic (CT) and ${ }^{18} \mathrm{~F}-\mathrm{NaF}$ positron emission tomographic (PET) imaging to unravel the mystery of why athletes have a high burden of coronary artery calcium (CAC), a strong predictor of cardiovascular morbidity and mortality, but have better survival than more sedentary persons. In their study, they found that " active", Apolipoprotein E deficient mice (Apo $\mathrm{E}^{-/-}$) have similar burdens of aortic CAC on microCT after 9 weeks of consuming a Western diet despite varying levels of activity, suggesting that exercise training did not alter plaque structure and may not modify risk. Taking their analysis a step further, however, they used ${ }^{18} \mathrm{~F}-\mathrm{NaF}$ PET imaging to examine the micro-architecture of the calcified plaque of "active" compared to "sedentary" mice. Interestingly, the mice randomized to exercise had a lower ${ }^{18} \mathrm{~F}-\mathrm{NaF}$ signal density, defined as ${ }^{18} \mathrm{~F}$ uptake normalized to injected dose per deposit volume, and each individual calcium deposit in the aorta had decreased mineral surface area index, calculated as the perimeter divided by the cross-sectional area by

Reprint requests: Patricia K. Nguyen, MD, Division of Cardiovascular Medicine, Stanford University, Stanford, CA 94305; pknguyen@stanford.edu

J Nucl Cardiol 2021;28:2215-9.

$1071-3581 / \$ 34.00$

Copyright (C) 2020 American Society of Nuclear Cardiology. histology. Taken together, these findings suggest that exercise reduces the amount of exposed surface area per unit bone volume, a measure that has been associated with plaque vulnerability. Based on the findings of Hsu et $\mathrm{al}^{1}{ }^{18} \mathrm{~F}-\mathrm{NaF}$ PET imaging may be a promising technology to better risk stratify patients and serially monitor the effectiveness of lifestyle modifications, such as exercise, or new pharmacological therapy designed to stabilize plaque.

\section{CORONARY ARTERY CALCIFICATION AS A MEASURE OF CARDIOVASCULAR RISK}

Coronary artery calcium (CAC) is a prominent feature of atherosclerosis, considered a form of ectopic bone formation. ${ }^{2}$ Now routinely measured using multidetector CT scanners, CAC has emerged as a widely available, consistent, and reproducible measure of cardiovascular risk. CAC is present in both men and women, both young and old, and across multiple ethnicities (e.g., whites, Asians, blacks, and Hispanics), ${ }^{3,4}$ representing a robust marker of atherosclerotic burden. Study after study has shown that CAC predicts cardiovascular risk beyond traditional risk factors, ${ }^{5-7}$ estimating the likelihood of suffering a cardiac event better than risk calculators such as the Framingham Risk Score, ACC/AHA ASCVD risk estimator, ${ }^{8}$ original MESA Risk Score Calculator, ${ }^{8}$ and Reynolds Risk Score. ${ }^{9}$ With such mounting evidence, not surprisingly, the American College of Cardiology has recently recommended that physicians use CAC to help reclassify patients with borderline to intermediate risk (10-year risk between $5 \%$ and $20 \%$ ) to guide the initiation of pharmacological therapy. ${ }^{10}$

For most of the patients, CAC has been shown to be a strong predictor of cardiovascular risk. A CAC score alone, however, may have limitations. Recent studies 


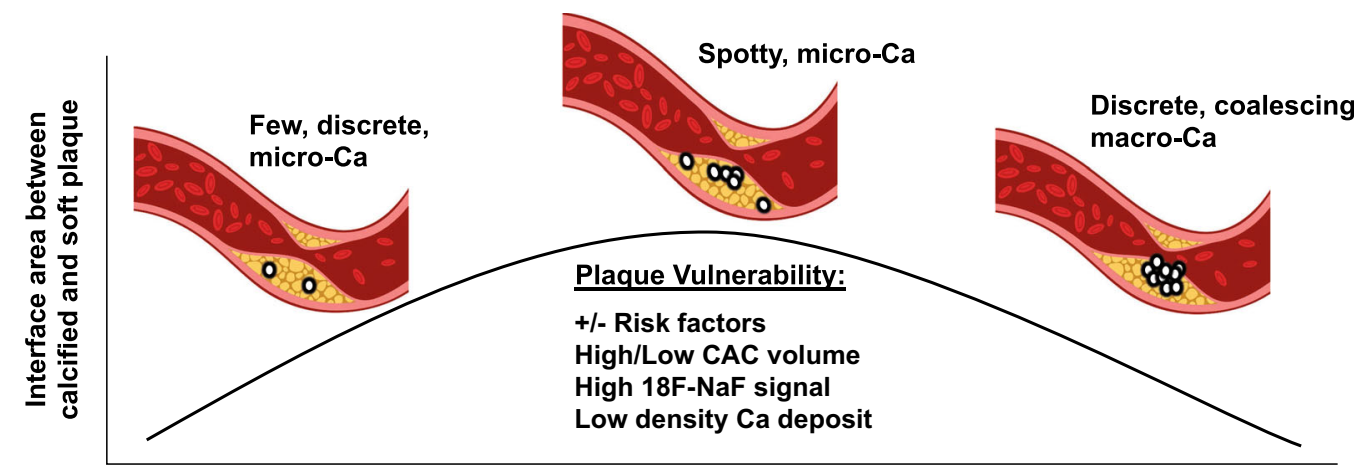

Figure 1. Schematic of the relationship between interface area and plaque vulnerability. Plaque vulnerability is difficult to predict. Patients with and without traditional risk factors suffer heart attacks. The relationship between coronary artery calcification (CAC) volume is also unreliable. Recent data suggests that $18 \mathrm{~F}-\mathrm{NaF}$ PET imaging and measurements of calcium $(\mathrm{Ca})$ density may be more reflective of plaque vulnerability because they measure the interface area between hard and soft plaque. Thus, plaques with spotty micro-calcification, which have high $18 \mathrm{~F}-\mathrm{NaF}$ signal and low density calcium may be most vulnerable to rupture.

have shown that some patients who are more physically fit, including marathon runners ${ }^{11}$ and athletes ${ }^{12,13}$ have higher levels of CAC than age-matched controls, but do not have increased cardiovascular morbidity and mortality. ${ }^{14}$ This clinical paradox suggest that measuring plaque burden alone is not enough to risk stratify all patients.

In their article, Hsu et $\mathrm{al}^{1}$ address this issue by randomizing Apo $\mathrm{E}^{-/-}$mouse, which develop aortic plaque after being fed a Western diet, to control vs an exercise training program with a rodent treadmill. After 9 weeks, they did not find a significant difference in coronary calcium burden between the two groups. Although these findings are consistent with clinical observations that plaque burden seems to be present whether you are active or sedentary, the lack of effect may result from an exercise regimen that was not intense enough or long enough to see these changes. Alternatively, their model may be sufficient, but we may need to look beyond overall plaque volume to determine how exercise can modify risk.

\section{IMAGING MORE THAN THE CAC VOLUME}

Multi-detector CTs can provide information not only on the total volume of calcium deposits in the coronary arteries to yield the CAC score, but also on calcium density, defined as the concentration of calcium present in a given atherosclerotic plaque. Interestingly, in contrast to CAC volume, a higher CAC density score, which is dependent on the highest Hounsfield units found in the plaque per given area, has been shown to be inversely associated with incident coronary heart disease for any CAC volume. ${ }^{15}$ Conversely, spotty calcification on $\mathrm{CT}$ is associated with $>2$-fold increase risk in plaque rupture (HR 2.25; 95\% CI 1.26-4.04; $P=0.006){ }^{16}$ Similarly, analysis of plaque vulnerability by intravascular ultrasound has revealed that patients with stable angina have fewer and larger contiguous plaques compared to those who present with acute coronary syndrome, who have a greater number of small calcium deposits. ${ }^{17}$ Taken together, these findings suggest that calcification may have a biphasic effect on plaque stability with early spotty deposition increasing plaque vulnerability and denser calcium deposition that group together into large areas stabilizing plaque (Figure 1).

It is likely that plaque rupture is caused by the failure to withstand the effects of fluid and mechanical stress, which occurs at the interface between materials of different stiffness. Specifically, at the interface between calcified and non-calcified plaque where the difference between stiffness can be as high as 4- to 5-fold, the plaque may be most vulnerable to longitudinal, circumferential and radial stress caused by pulsatile blood coursing through the arteries. ${ }^{18}$ Based on the principals of biomechanics, it appears that how and where calcium deposits interface with other components of plaque are important determinants of plaque vulnerability. In other words, the degree of plaque vulnerability is proportional to the interface area between calcified and non-calcified plaque, which decreases as the calcification areas continue to form, grow, and coalesce. Taking this into account, perhaps a more valuable measure for predicting plaque vulnerability may be to measure the interface surface area between calcified and non-calcified plaque rather than the overall calcium burden, as measured by the CAC score. Although calcium density can approximate areas where calcium deposits are tightly grouped 
together, it does not reflect the surface area between calcified and soft plaque that is exposed to stressors that can lead to rupture. Another measure, thus, may be warranted.

First introduced as a tracer for imaging skeletal disorders in 1962 and FDA approved since $1972,{ }^{18} \mathrm{~F}$ sodum fluoride $\left({ }^{18} \mathrm{~F}-\mathrm{NaF}\right)$ has emerged as a promising vascular imaging agent that can directly image active calcification and quantify areas of microcalcification. In bone, ${ }^{18} \mathrm{~F}-\mathrm{NaF}$ incorporates into exposed areas of hydroxyapatite, and, thus, may be able to image active bone remodeling. In the vasculature, ${ }^{18} \mathrm{~F}-\mathrm{NaF}$ binds to micro-calcifications and to the outer surface, but not inner surfaces of macrocalcifications, suggesting it may be a good measure of the interface between calcified plaque and non-calcified plaque. ${ }^{19,20}$ Importantly, a prospective clinical trial showed that increased ${ }^{18} \mathrm{~F}$ $\mathrm{NaF}$ uptake was associated with vulnerable plaques, which was verified by histology ex vivo in the case of carotid plaques and by intravascular ultrasound in vivo with respect to coronary plaques. ${ }^{21}$

To complement their findings on $\mathrm{CT}$ and to examine the plaque micro-architecture, Hsu et al ${ }^{1}$ used ${ }^{18} \mathrm{~F}-\mathrm{NaF}$ PET imaging to measure the exposed surface area of calcium deposits before and after exercise training. They found that the PET signal density (percent injected dose of tracer per deposit volume) but not the total surface area (percent injected dose) was significantly reduced after exercise. Because ${ }^{18} \mathrm{~F}-\mathrm{NaF}$ also binds to the surface of macrocalcifications, which may actually increase with exercise and improve plaque stability, these results are not surprising. Using histology to verify their ${ }^{18} \mathrm{~F}-\mathrm{NaF}$ PET findings, they also found that the mineral area surface index, the total perimeter divided by the total cross-sectional area, measured per histological section, decreased with exercise, which is consistent with the changes in signal density on PET imaging. By imaging more than CAC volume, Hsu et $\mathrm{al}^{1}$ have presented a plausible mechanism by which athletes can have high levels of CAC but have a low incidence of cardiovascular events. Based on their model, the overall burden of plaque may be dependent on the Western diet, but how and where plaques develop, either as a few, discrete dense plaques or spotty, micro-calcifications, is likely shaped by physical activity levels.

\section{TRANSLATIONAL IMPLICATIONS}

Accurately assessing cardiovascular risk has been the holy grail of preventative cardiology. While risk estimate calculators, all of which are based on pooled cohorts including information on age, sex, race, and traditional risk factors, have proven invaluable in guiding preventative care, they have over- and under-estimated risk in certain subpopulations including patients with a family history of premature cardiovascular disease, women, South Asians, and other Asians. ${ }^{22}$ To address this limitation, some risk calculators have added a measure of inflammation and the CAC score, which have resulted in improved accuracy. ${ }^{23,24}$ An assessment of physical fitness and physical activity, however, is still missing despite ample evidence that these "vital signs" are better predictors of cardiovascular morbidity and mortality than traditional risk factors. ${ }^{25-28}$

This brings us to the following question: How can we accurately assess the risk of an active individual or elite athlete who has a CAC score $>400$ ? Placing the patient on high intensity statin may be an option but this can expose these patients to the risk of statin-related myopathy, hepatotoxicity, glucose intolerance, and potentially memory loss. Depending on the location of the calcifications, the patient may have anxiety about these findings and wonder if he/she should have a cardiac CT angiography or stress imaging to evaluate whether they need a diagnostic cardiac catheterization. Lesions located in the left main or proximal left anterior

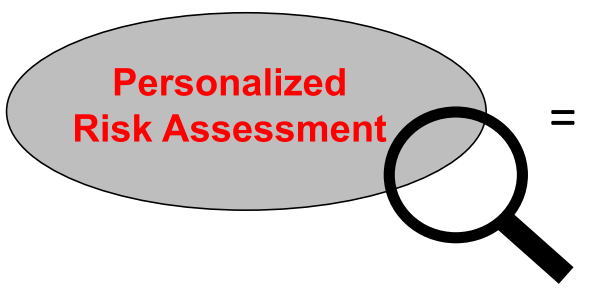

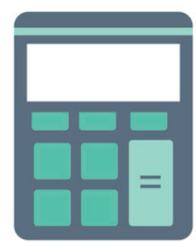

Risk

Calculator

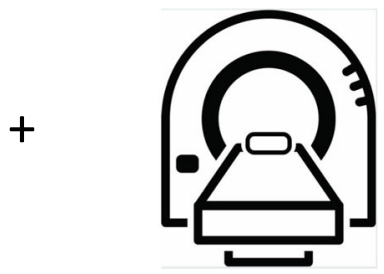

PET/CT of Plaque Macroand Micro-Architecture

Figure 2. Schematic of how to improve risk stratification. To deliver a more personalized approach toward risk stratification, we need to incorporate risk calculators that are sex- and racespecific and include measures of physical activity and fitness. Moreover, the addition of imaging to closely evaluate the macro- and micro-architecture would help to identify the most vulnerable patients. 
descending artery can create even more consternation. Despite the presence of calcium deposits, which can often cause a blooming artifact on CT and make lesions appear larger than they are, the likelihood is low that the patient has significant obstruction warranting an intervention if he is physically active, fit, and asymptomatic. Based on recent findings from the International compariSon of comparative Health Effectiveness with Medical and Invasive Approaches (ISCHEMIA) trial, revascularization will also not reduce the risk of morbidity and mortality if the patient does not have angina or an anginal-equivalent.

The physically fit person with a high plaque burden based on their CAC score, however, may benefit from further characterization of their plaque micro-architecture. Although not ready for prime time, ${ }^{18} \mathrm{~F}-\mathrm{NaF}$ PET imaging can potentially help risk stratify these patients. ${ }^{29}$ The absence of ${ }^{18} \mathrm{~F}-\mathrm{NaF}$ PET signal in calcified lesions likely indicates stable plaque. Further validation with large multi-center randomized trials, however, are needed to determine the negative predictive value of ${ }^{18} \mathrm{~F}-\mathrm{NaF}$ PET imaging. While the presence of ${ }^{18} \mathrm{~F}-\mathrm{NaF}$ PET uptake may indicate plaque vulnerability, it would be important to correlate the amount of signal density with the degree of risk in different patient populations and in patients with varying levels of plaque burden. Importantly, it seems that ${ }^{18} \mathrm{~F}-\mathrm{NaF}$ PET imaging is sensitive enough to pick up changes after lifestyle interventions like exercise, but its accuracy and reproducibility need further study. Finally, the mechanisms by which calcium deposits in atherosclerotic plaque take up ${ }^{18} \mathrm{~F}-\mathrm{NaF}$ should be investigated to refine this technology. Although our current risk stratification tools are still imperfect because of our reliance on epidemiological data, we continue to make strides in our ability to image the vulnerable plaque, so that one day we can deliver more personalized approaches to preventative care (Figure 2).

Calcification may have a biphasic effect on plaque stability. Early spotty deposition will increase the surface area between calcified and non-calcified plaque, which increases exposure to mechanical stress and, thus, making the plaque vulnerable to rupture. As the calcification deposits grows, becomes denser, and coalesces, the interface between calcified plaque and non-calcified plaque decreases and the plaque becomes more stable. It remains unclear how traditional risk factors affect this process although exercise appears to decrease the surface area to volume ratio.

\section{Disclosure}

Xianxi Huang, Jessica D'Addabbo and Patricia K. Nguyen have no disclosures.

\section{References}

1. Hsu JJ, Fong F, Patel R, et al. Changes in microarchitecture of atherosclerotic calcification assessed by ${ }^{18} \mathrm{~F}-\mathrm{NaF}$ PET and CT after a progressive exercise regimen in hyperlipidemic mice. $\mathrm{J}$ Nucl Cardiol 2020. https://doi.org/10.1007/s12350-019-02004-3.

2. Tintut Y, Alfonso Z, Saini T, et al. Multilineage potential of cells from the artery wall. Circulation 2003;108:2505-10.

3. McClelland RL, Chung H, Detrano R, Post W, Kronmal RA. Distribution of coronary artery calcium by race, gender, and age: results from the Multi-Ethnic Study of Atherosclerosis (MESA). Circulation 2006;113:30-7.

4. McClelland RL, Jorgensen NW, Budoff M, et al. 10-year coronary heart disease risk prediction using coronary artery calcium and traditional risk factors: derivation in the MESA (Multi-Ethnic Study of Atherosclerosis) with validation in the HNR (Heinz Nixdorf Recall) study and the DHS (Dallas Heart Study). J Am Coll Cardiol 2015;66:1643-53.

5. Kavousi M, Desai CS, Ayers C, et al. Prevalence and prognostic implications of coronary artery calcification in low-risk women: A meta-analysis. JAMA 2016;316:2126-34.

6. Yano Y, O'Donnell CJ, Kuller L, et al. Association of coronary artery calcium score vs age with cardiovascular risk in older adults: An analysis of pooled population-based studies. JAMA Cardiol 2017;2:986-94.

7. Ferencik M, Pencina KM, Liu T, et al. Coronary artery calcium distribution is an independent predictor of incident major coronary heart disease events: Results from the Framingham heart study. Circ Cardiovasc Imaging 2017;10:e006592.

8. Nasir K, Bittencourt MS, Blaha MJ, et al. Implications of coronary artery calcium testing among statin candidates according to American College of Cardiology/American Heart Association Cholesterol Management Guidelines: MESA (Multi-Ethnic Study of Atherosclerosis). J Am Coll Cardiol 2015;66:1657-68.

9. Alashi A, Lang R, Seballos R, et al. Reclassification of coronary heart disease risk in a primary prevention setting: Traditional risk factor assessment vs. coronary artery calcium scoring. Cardiovasc Diagn Ther 2019;9:214-20.

10. Arnett DK, Blumenthal RS, Albert MA, et al. 2019 ACC/AHA guideline on the primary prevention of cardiovascular disease: executive summary: A report of the American College of Cardiology/American Heart Association Task Force on clinical practice guidelines. J Am Coll Cardiol 2019;74:1376-414.

11. Mohlenkamp S, Lehmann N, Breuckmann F, et al. Running: the risk of coronary events: Prevalence and prognostic relevance of coronary atherosclerosis in marathon runners. Eur Heart $\mathrm{J}$ 2008;29:1903-10.

12. Merghani A, Maestrini V, Rosmini S, et al. Prevalence of subclinical coronary artery disease in masters endurance athletes with a low atherosclerotic risk profile. Circulation 2017;136:126-37.

13. Aengevaeren VL, Mosterd A, Braber TL, et al. Relationship between lifelong exercise volume and coronary atherosclerosis in athletes. Circulation 2017;136:138-48.

14. DeFina LF, Radford NB, Barlow CE, et al. Association of allcause and cardiovascular mortality with high levels of physical activity and concurrent coronary artery calcification. JAMA Cardiol 2019;4:174-81.

15. Criqui MH, Denenberg JO, Ix JH, et al. Calcium density of coronary artery plaque and risk of incident cardiovascular events. JAMA 2014;311:271-8.

16. Nerlekar N, Ha FJ, Cheshire C, et al. Computed tomographic coronary angiography-derived plaque characteristics predict major adverse cardiovascular events: A systematic review and metaAnalysis. Circ Cardiovasc Imaging 2018;11:e00697. 
17. Ehara S, Kobayashi Y, Yoshiyama M, et al. Spotty calcification typifies the culprit plaque in patients with acute myocardial infarction: An intravascular ultrasound study. Circulation 2004;110:3424-9.

18. Richardson PD, Davies MJ, Born GV. Influence of plaque configuration and stress distribution on fissuring of coronary atherosclerotic plaques. Lancet 1989;2:941-4.

19. Irkle A, Vesey AT, Lewis DY, et al. Identifying active vascular microcalcification by (18)F-sodium fluoride positron emission tomography. Nat Commun 2015;6:7495.

20. Dweck MR, Chow MW, Joshi NV, et al. Coronary arterial $18 \mathrm{~F}$ sodium fluoride uptake: A novel marker of plaque biology. J Am Coll Cardiol 2012;59:1539-48.

21. Joshi NV, Vesey AT, Williams MC, et al. 18F-fluoride positron emission tomography for identification of ruptured and high-risk coronary atherosclerotic plaques: A prospective clinical trial. Lancet 2014;383:705-13.

22. Damen JA, Pajouheshnia R, Heus P, et al. Performance of the Framingham risk models and pooled cohort equations for predicting 10-year risk of cardiovascular disease: A systematic review and meta-analysis. BMC Med 2019;17:109.

23. Cook NR, Paynter NP, Eaton CB, et al. Comparison of the Framingham and Reynolds Risk scores for global cardiovascular risk prediction in the multiethnic Women's Health Initiative. Circulation 2012;125:1748-56.
24. Greenland P, Blaha MJ, Budoff MJ, Erbel R, Watson KE. Coronary calcium score and cardiovascular Risk. J Am Coll Cardiol 2018;72:434-47.

25. Myers J, Prakash M, Froelicher V, Do D, Partington S, Atwood JE. Exercise capacity and mortality among men referred for exercise testing. N Engl J Med 2002;346:793-801.

26. Myers J, Kaykha A, George S, et al. Fitness versus physical activity patterns in predicting mortality in men. Am J Med 2004;117:912-8.

27. Gulati M, Pandey DK, Arnsdorf MF, et al. Exercise capacity and the risk of death in women: The St James Women Take Heart Project. Circulation 2003;108:1554-9.

28. Ross R, Blair SN, Arena R, et al. Importance of assessing cardiorespiratory fitness in clinical practice: A case for fitness as a clinical vital sign: a scientific statement from the American Heart Association. Circulation 2016;134:e653-99.

29. Bellinge JW, Francis RJ, Majeed K, Watts GF, Schultz CJ. In search of the vulnerable patient or the vulnerable plaque: (18)Fsodium fluoride positron emission tomography for cardiovascular risk stratification. J Nucl Cardiol 2018;25:1774-83.

Publisher's Note Springer Nature remains neutral with regard to jurisdictional claims in published maps and institutional affiliations. 